\title{
High Performance Activated Carbon Paper-Based electrode for Microbial Fuel Cells
}

\author{
Kenta Sato ${ }^{1}$, Dang Trang Nguyen ${ }^{1}$, Daiki Fujita ${ }^{2}$, Ryuta Tobe ${ }^{2}$, Hisaaki Mihara ${ }^{2}$ and Kozo Taguchi ${ }^{1}$ \\ ${ }^{1}$ Department of Electrical and Electronic Engineering, Ritsumeikan University, Kusatsu, Shiga, Japan \\ ${ }^{2}$ Department of Biotechnology, College of Life Sciences, Ritsumeikan University, Kusatsu, Shiga, Japan
}

Correspondence Author: Kozo Taguchi, Department of Electrical and Electronic Engineering, Ritsumeikan University, 1-1-1 Noji-Higashi, Kusatsu, Shiga 5258577, Japan,

Email: taguchi@se.ritsumei.ac.jp

Received date: 22 January 2019, Accepted date: 14 April 2019, Online date: 25 April 2019

Copyright: (c) 2019 Kenta Sato, Dang Trang Nguyen, Daiki Fujit, Ryuta Tobe, Hisaaki Mihara and Kozo Taguchi.2019. This is an open-access article distributed under the terms of the Creative Commons Attribution License, which permits unrestricted use, distribution, and reproduction in any medium, provided the original author and source are credited.

\begin{abstract}
Microbial fuel cell (MFC) has been paid attentions as a new renewable energy resource. This study considered the evaluation of activated carbon (AC) paper as an anode and cathode electrode material for MFC. AC has novel properties that possesses a large specific surface area, a large amount of micropores and mesopores, low inner resistance and high biofilm adhesion. In this study, AC paper and hydrophilic carbon paper (CP) were prepared as the anode electrodes. The performance of the AC paper was compared to the hydrophilic CP in terms of methylene blue (MB) adsorption capacity, Escherichia coli (E. coli) adsorption capacity, scanning electron microscope (SEM) images and power density characteristics. The produced maximum power density of $11.1 \mu \mathrm{W} / \mathrm{cm}^{2}$ was generated by the AC paper, which is much greater than that of the hydrophilic CP. Moreover, we achieved $43 \%$ improvement in power density when using AC paper with larger thickness as cathode electrode. These results attributed to a large surface area and low inner resistance of the AC paper. The large surface area promoted biofilm growth and made electrochemical reduction reaction better, which enhanced the generation of power output.
\end{abstract}

Keywords: Microbial fuel cell renewable energy resource activated carbon paper

\section{INTRODUCTION}

Recently, we have been faced a lot of energy problems such as oil and coal depletion and carbon dioxide emissions due to massconsumption of fossil fuel while developing an alternative renewable and environmentally friendly energy sources have been paid attention all over the world[1]. Microbial fuel cell (MFC) is one of the alternative promising energy resources[2]. MFC is capable of converting chemical energy into electrical energy directly[3].

Mainly, there are two kinds of MFC configuration, which are double chamber type and single chamber type. A double chamber MFC consists of two chambers which are separated by proton exchange membrane (PEM). Microorganism produces electrons, protons and $\mathrm{CO}_{2}$ by decomposing organic substrates. The produced electrons are transferred from anode chamber to the cathode chamber through an external circuit, meantime, and the produced protons are passed thorough the PEM to cathode chamber. The electrons and protons transferred to cathode combined with oxygen in the air to form water with oxidizing agent[4][5]. On the other hand, a single chamber MFC has no cathode chamber, so cathode is directly exposed to the air. In this study, the doublechamber MFC is used. Although MFC is affordable and promising energy resources, power generation efficiency is relatively low $[5]$.

Currently, a lot of investigations for MFC such as the material of electrodes, use of mediator, membrane and cell construction have been conducted to improve the performance.[6] The characteristics of the anode electrodes play an important role to boost power generation because it has closely relationship between electron transfer and the microorganism. [6] Carbon-based electrodes including carbon paper, carbon brush, carbon cloth, and graphite have been commonly used as anode electrode in MFC due to good conductivity and low costs, and their evaluation has widely reported. [2] 
Activated carbon (AC) is a novel and promising material because of the high adsorption capacity of both gases and solutes. Thus, it has been used for sewage purification, removal of organic pollutants, etc. [7] The use of that has been applied to various fields including purification of industrial waste, the automobile industry and the household utensils while it is effective as an anode electrode material in MFC. [S. S. Manickam] concluded that activated carbon nanofiber nonwovens with a large surface area and open porous structure produced higher power generation compare to carbon cloth and granular activated carbon. They reported that a large surface area that AC has helps formation of biofilm and it links with power generation closely. [8]

AC is made from various carbonaceous substances such as coal, coconut shells, sawdust, and wood chips. [9] The process to fabricate AC is divided into two methods, which are called physical activation and chemical activation. For Physical activation, the char obtained by carbonization of the carbonaceous substances is gasified by a physical activating agent such as steam and carbon dioxide at a temperature in the range of $700-900^{\circ} \mathrm{C}$. For Chemical activation, woody raw materials like a sawdust are used. The precursor is firstly impregnated with a chemical agent such as zinc chloride, phosphoric acid, KOH, then the impregnated material is carbonized under an inert atmosphere. Finally, the carbonized material is washed to remove the chemical.

In this study, a simple paper-based electrode was used. The performance of the AC paper was compared to hydrophilic CP from various methods such as methylene blue (MB) adsorption capacity, Escherichia coli (E. coli) adsorption, scanning electron microscope (SEM) images and power density characteristics in MFC. Moreover, we tried to improve the power density by increasing the thickness of the cathode electrode.

\section{MATERIAL AND METHODS}

\subsection{MFC configuration (construction)}

The detail of MFC construction used in this study is shown in Fig. 1. A double chamber MFC made of acryl transparent board was constructed. The volumes of both the anode chamber and cathode chamber were $9 \mathrm{~cm}^{2}(1.0 \mathrm{~cm} \times 3.0 \mathrm{~cm} \times 3.0 \mathrm{~cm})$. Area of PEM was $3 \mathrm{~cm}^{2}(1.5 \mathrm{~cm} \times 2.0 \mathrm{~cm})$. The hydrophilic CP $(\mathrm{C} 1 \mathrm{P}-2$, AZUMI FILTER PAPER CO., LTD.) and the AC paper (C1P1AC1, AZUMI FILTER PAPER CO., LTD.) were used as the anode electrodes, while two kinds of AC paper with thicknesses of $0.23 \mathrm{~mm}$ (C1P1AC1, AZUMI FILTER PAPER CO., LTD.) and 0.35mm (RCA-01, AZUMI FILTER PAPER CO., LTD.) were used as the cathode electrodes. Characteristics of each electrode were shown in Table1. Both areas of anode electrode and cathode electrode were $5.25 \mathrm{~cm}^{2}(1.5 \mathrm{~cm} \times 3.5 \mathrm{~cm})$ and hydrophobic CP $(\mathrm{C} 1 \mathrm{E}-1$, AZUMI FILTER PAPER CO., LTD.) was attached to each top of the electrodes with a plastic clip to prevent electric clip from oxidation. The area of hydrophobic CP was $4.5 \mathrm{~cm}^{2}(1.5 \mathrm{~cm} \times 3.0 \mathrm{~cm})$.

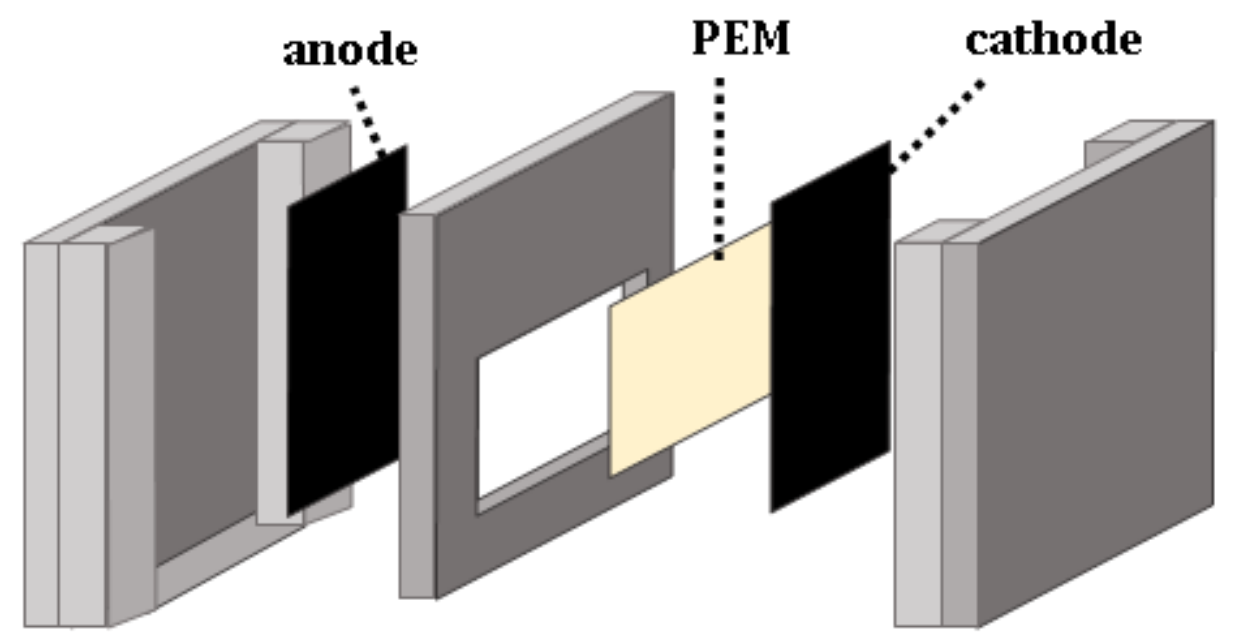

Fig.1. MFC configuration

Table 1: Properties of AC paper and CP

\begin{tabular}{|c|c|c|c|c|c|}
\hline Samples & Compounding ratio (\%) & Paper weight $\left(\mathbf{g} / \mathbf{m}^{2}\right)$ & Thickness $(\mathbf{m m})$ & Density $(\mathbf{g} / \mathbf{c m})$ & Resistivity $(\mathbf{m} \Omega$-cm) \\
\hline C1P1AC1 & $\begin{array}{c}\text { Activated carbon: 60 } \\
\text { Carbon fiber: 20 } \\
\text { Polyacrylonitrile fiber: 20 }\end{array}$ & 51 & 0.23 & 0.24 & 152 \\
\hline RCA-01 & $\begin{array}{c}\text { Activated carbon: 60 } \\
\text { Carbon fiber: 20 } \\
\text { Polyacrylonitrile fiber: 20 }\end{array}$ & 112 & 0.35 & 0.32 & 203 \\
\hline
\end{tabular}




\begin{tabular}{|l|c|c|c|c|c|}
\hline C1P-2 & $\begin{array}{c}\text { Carbon fiber: 50 } \\
\text { Cellulose fiber: 50 }\end{array}$ & 51 & 0.26 & 0.20 & 109 \\
\hline
\end{tabular}

\subsection{Growth curve of Escherichia coli}

In this study, E.coli was used as biocatalyst because it is accessible to culture [3]. Before MFC experiments, the time that is required until the number of bacteria becomes stable was measured and growth curve of $E$. coli was obtained. Optical density was measured every two hours (h) at 590nm (OD590) with colourimeter (Colourwave CO7500 Colorimeter, Funakoshi) after the inoculation into a 40ml Lysogeny Broth (LB) medium that was composed of $10 \mathrm{~g} / 1$ Tryptone, $5 \mathrm{~g} / \mathrm{l} \mathrm{Yeast} \mathrm{extract,} 10 \mathrm{~g} / \mathrm{l} \mathrm{NaCl}$ and $\mathrm{NaOH}$ to adjust $\mathrm{PH}=7.0$. The $\mathrm{LB}$ solution was put in shaking incubator at $30^{\circ} \mathrm{C}$ for 72 hours.

\subsection{Biofilm formation on anode electrodes materials}

The AC paper and hydrophilic CP were respectively put into a cup contained $40 \mathrm{ml} \mathrm{LB}$ solution that had been conducted shaking culture at $30^{\circ} \mathrm{C}$ for 2 days. Moreover, these papers were set up vertically to the surface of the solution by using a paper stand. It was made of acryl transparent board (Fig. 2). This setup method is expected to prevent waste matters that were discharged by bacteria and the dead bodies of bacteria from attaching on the surface of these AC papers. Subsequently, biofilm was formed on these $\mathrm{AC}$ papers in incubator at $30^{\circ} \mathrm{C}$ for 24 hours.

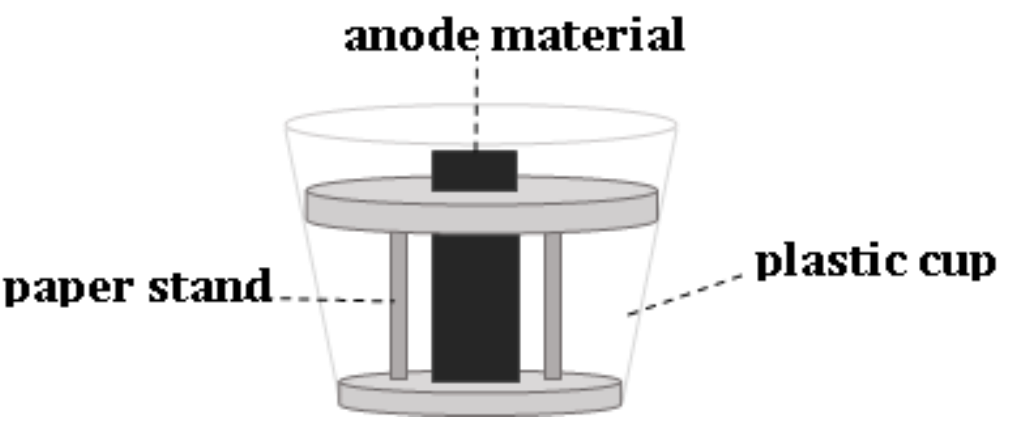

Fig.2. Design of the paper stand for growing biofilm on the anode electrode

\subsection{Scanning electron microscope images}

The biofilms formed on the AC paper and hydrophilic CP were observed using a scanning electron microscope (SEM, S-4300 Hitachi). Some processes were conducted before measuring by SEM. Firstly, these biofilm-loaded papers were immersed in the sterilizer solution to fix the bacterial cells and they were put in a refrigerator overnight. Secondly, they were immersed in four kinds of different concentration of ethanol solutions of 50, 70, 90, 100\% continuously and they were naturally dried. Lastly, they were sputter-coated with Au using a quick coater (VPS-020, ULVAC).

\subsection{E. coli adsorption measurement}

E. coli was stationary cultured in $2 \mathrm{~mL}$ of $\mathrm{LB}$ medium in microtubes, and the microtubes was put in an incubator at $30{ }^{\circ} \mathrm{C}$ for 48 hours. The cultured solution was centrifuged and the LB medium was discarded, and then dilution was conducted using physiological saline to equal the number of $E$. coli in respective microtubes and to inhibit further growth of $E$. coli . It was proved that E. coli cannot proliferate in physiological saline. Next, AC paper and hydrophilic CP of $0.5 \mathrm{~cm} 2(0.5 \mathrm{~cm} \times 1.0 \mathrm{~cm})$ were respectively added in the microtubes. These microtubes were put in an incubator at $30{ }^{\circ} \mathrm{C}$. OD600 of these solutions was measured immediately after the papers were added and after 24 hours, respectively.

\subsection{Methylene blue adsorption measurement}

The adsorption capacity of Methylene blue (MB) on two kinds of AC paper with thicknesses of $0.23 \mathrm{~mm}$ and $0.35 \mathrm{~mm}$ and hydrophilic CP were measured at $680 \mathrm{~nm}$ with the colourimeter. AC paper and hydrophilic $\mathrm{CP}$ of $0.5 \mathrm{~cm} 2(0.5 \mathrm{~cm} \times 1.0 \mathrm{~cm})$ were respectively added in microtubes contained $2 \mathrm{ml}$ of $0.2 \mathrm{mM} \mathrm{MB}$ solution. Firstly, the optical absorbance of MB was measured immediately after these papers were added and after 24 hours. These papers were left in the microtube during the measurement.

\subsection{MFC operation}

The MFC was operated in batch mode. The anode chamber was filled with $6 \mathrm{ml}$ medium composed of $10 \mathrm{~g} / 1 \mathrm{glucose}, 5 \mathrm{~g} / \mathrm{l}$ yeast extract, $10 \mathrm{~g} / 1 \mathrm{NaHCO}_{3}$, and $8.5 \mathrm{~g} / 1 \mathrm{NaH}_{2} \mathrm{PO}_{4}$. While the cathode chamber was filled with $6 \mathrm{ml}$ potassium ferricyanide solution of 
$50 \mathrm{mM}$. The biofilm-loaded AC paper and hydrophilic CP were used as the anode electrodes, and two kinds of AC paper with different thicknesses were used as the cathode electrodes.

\subsection{Power density measurement in MFCs}

The voltage value across the resistance was measured every 20 seconds with a data acquisition system (DAQ, National Instrument). The current density and power density were calculated by the voltage value obtained from this experiment. Both electrodes were put into each chamber where the immerged surface areas were one-third of the surface areas of the electrodes. Hence, the current density was obtained by dividing the present to the actual surface area of $3 \mathrm{~cm}^{2}(1.5 \mathrm{~cm} \times 2.0 \mathrm{~cm})$.

In this study, the following two experiments were conducted.

(1) Power density measurement using AC paper and CP as the anode electrodes

In the case of using $\mathrm{AC}$ paper, a $2.7 \mathrm{k} \Omega$ resister was first connected to the external circuit, and then the resister was changed to lower resistance $(1.8 \mathrm{k} \Omega, 1 \mathrm{k} \Omega, 680 \Omega, 470 \Omega$, and $270 \Omega)$ to obtain the polarization curve and power density curve. In the case of using hydrophilic $\mathrm{CP}$, a $68 \mathrm{k} \Omega$ resister was first connected to the external circuit, and then the resister was changed to lower resistance $(46 \mathrm{k} \Omega, 18 \mathrm{kk} \Omega, 10 \mathrm{k} \Omega, 6.7 \mathrm{k} \Omega, 4.7 \mathrm{k} \Omega, 2.7 \mathrm{k} \Omega$, and $1 \mathrm{k} \Omega)$.

(2) Power density measurement using two kinds of AC paper with the thickness of $0.23 \mathrm{~mm}$ and $0.35 \mathrm{~mm}$ as the cathode electrodes

In the case of using either AC papers with the thickness of $0.23 \mathrm{~mm}$ and $0.35 \mathrm{~mm}$, a $2.7 \mathrm{k} \Omega$ resister was first connected to the external circuit, and then the resister was changed to lower resistance $(1.8 \mathrm{k} \Omega, 1 \mathrm{k} \Omega, 680 \Omega, 470 \Omega$, and $270 \Omega)$ to obtain the polarization curve and power density curve.

\section{RESULT AND DISCUSSION}

\subsection{Growth curve of E. coli}

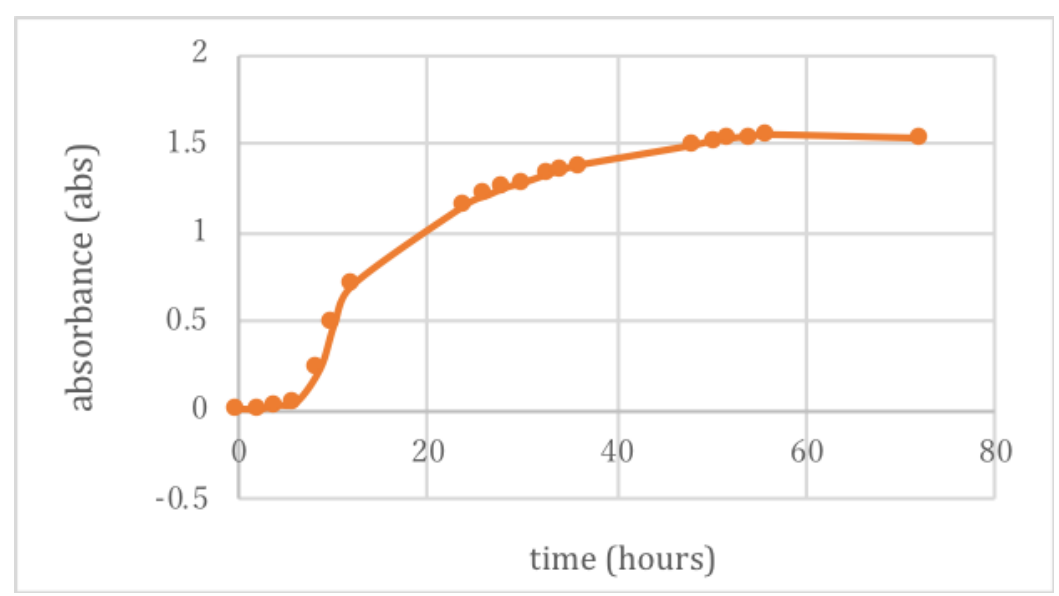

Fig.3. Growth curve of $E$. coli in a LB medium for 72 hours.

A growth curve of $E$. coli in a $40 \mathrm{ml} \mathrm{LB}$ medium was obtained as shown in Fig. 3. The data shows that the number of bacteria in a LB solution hardly changed from 0 to 8 hours and sharply increased from 8 to 48hours and became stable after 48 hours. These periods are respectively called 'lag phase', 'log phase', 'stationary phase'. During a lag phase, bacteria do not undergo cell division and adapt themselves to LB medium. During a log phase, bacteria begin to undergo cell division and the number of cells increases sharply. During a stationary phase, Growth rate and death rate become equal due to depletion of nutrients and accumulation of inhibitory matters. Therefore, the AC paper and hydrophilic CP were put into LB medium after 48 hours shaking culture.

\subsection{SEM images}



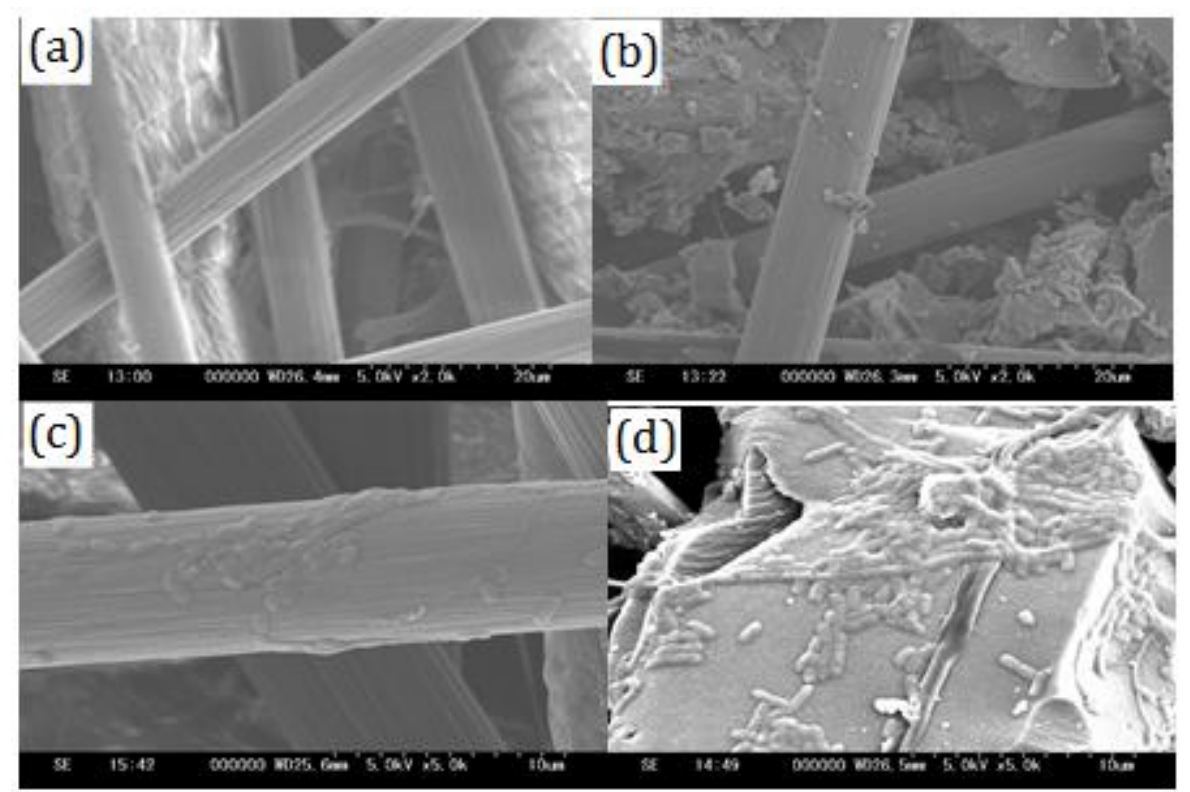

Fig. 4. SEM images of original CP and AC papers (a, b). (c, d) show images of the formed biofilm on the surface of the CP and AC paper

Fig. 4 (a, b) shows the surface of the original hydrophilic $\mathrm{CP}$ and AC paper. These images were obtained at the magnification of $20 \mu \mathrm{m}$. Fig. 4 (c, d) shows the biofilm formed on the surface of hydrophilic CP and AC paper. These images were obtained at the magnification of $10 \mu \mathrm{m}$. As seen in (a), there are a lot of spaces between carbon fibers because hydrophilic CP is composed of carbon fiber and binder material only. As seen in Fig. (b), AC powder was packed very carefully between carbon fibers and which extended the surface area. As seen in Fig 4 (c), a few biofilms were scattered on the carbon fibers while the biofilms are barely seen on the binder material. As seen in Fig. 4 (d), there were some biofilms not only on the carbon fibers but also on the AC powders. Moreover, the biofilm could be seen on the AC powders more frequently than on the carbon fibers because a lot of micropores and mesopores on the surface of AC powders which help biofilm adhesion. Consequently, the biofilm formation on the AC paper was better than that of the hydrophilic CP. It was indicated that a large surface area promotes biofilms formation.

\subsection{E. coli adsorption measurement}

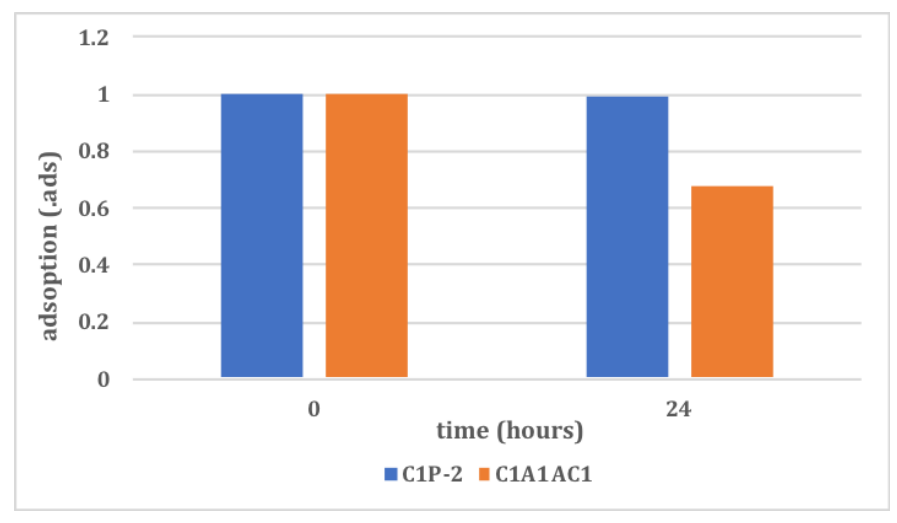

Fig. 5. E. coli absorbance value of AC papers and hydrophilic carbon paper

This experiment quantitatively showed the capacity of adsorption of $E$. coli by the electrode materials. CP hardly adsorbed $E$. coli, while AC paper adsorbed $E$. coli well. Seen from the difference in the adsorption value of $E$. coli, it is presumed that AC paper is more effective to form a biofilm.

\subsection{Methylene blue adsorption measurement}




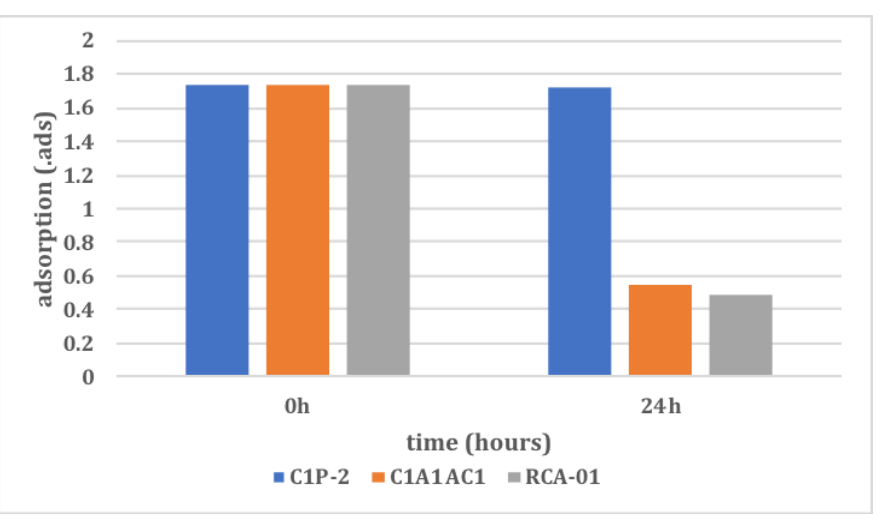

Fig. 6. MB absorbance value of AC papers and hydrophilic carbon paper

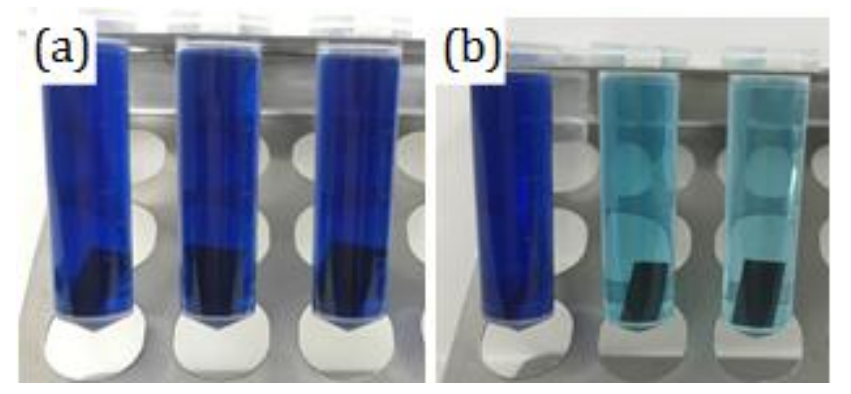

Fig. 7. Photo images of MB adsorption measurement. (a) photo image was taken immediately after the papers were added. (b) photo image was taken after 24 hours of adding the documents

Fig. 6 and Fig. 7 shows MB absorbance changed in 24 hours. MB adsorption value with AC papers decreased sharply, while hydrophilic CP barely changed. Therefore, it was indicated that AC powders included in AC paper plays an essential role to adsorb MB. AC paper with thickness of $0.35 \mathrm{~mm}$ had little higher adsorbing performance than AC paper with a thickness of $0.23 \mathrm{~mm}$, because of little larger surface area. MB has an effective molecule diameter of about 1.5nm, thus the MB adsorption capacity of AC related to the amount of specific surface area which exists in pores of this size and greater in diameter. AC made from coconut shell by physical activation has maximum pore size distribution with pore width around $1.1 \mathrm{~nm}$, however it has the broad pore size distribution in the range of from about 0.5 to $4.0 \mathrm{~nm}$. Therefore, a lot of micropores and mesopores which are larger than effective molecule diameter of $\mathrm{MB}$ is the crucial component to adsorb MB.

\subsection{Power density measurement in the MFCs}

(1) Power density measurement using AC paper and CP as the anode electrodes

The discharging voltage and the power density as a function of current frequency are shown in Fig. 8 and Fig. 9, respectively. It was proved that the internal resistance of the MFC corresponding to the external resistor where the maximum power output is obtained. Therefore, the internal resistance of the MFCs using the AC paper was estimated about $680 \Omega$ and that of the case using the hydrophilic CP was estimated about $18 \mathrm{k} \Omega$ (Fig. 9). Low internal resistance contributes to the improvement of the power output. Thus, as shown in Fig. 8 and Fig. 9, the MFC with the hydrophilic CP electrode generated higher open-circuit voltage than the case using AC paper electrode, however power density was much lower due to high internal resistance. As shown in Fig. 9, the AC paper generated the maximum power density of $11.1 \mu \mathrm{W} / \mathrm{cm}^{2}\left(73.8 \mu \mathrm{A} / \mathrm{cm}^{2}\right)$, which was about 36 times higher than the maximum power density generated by the hydrophilic CP. This is because of that a large surface area of the AC paper promoted to form more bacterial biofilm on the anode electrode, which enhanced the power density. 


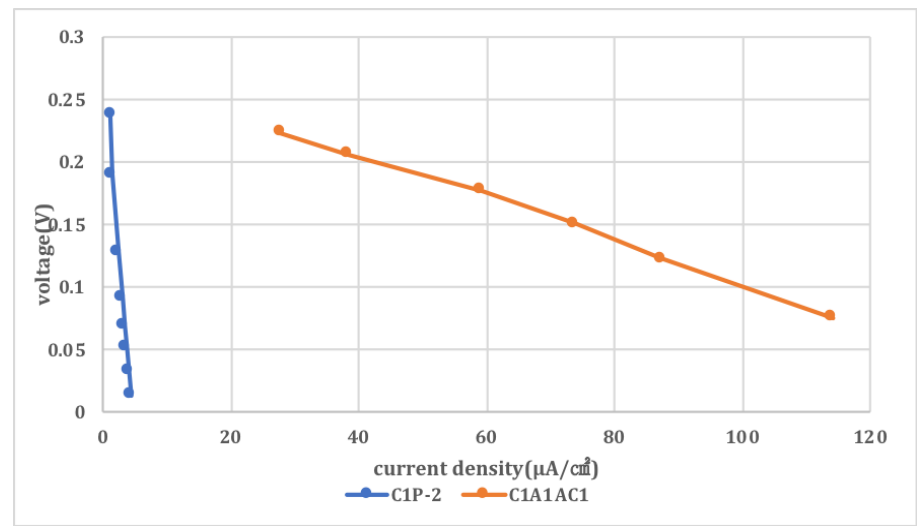

Fig. 8. Voltage and current density generated by the MFCs

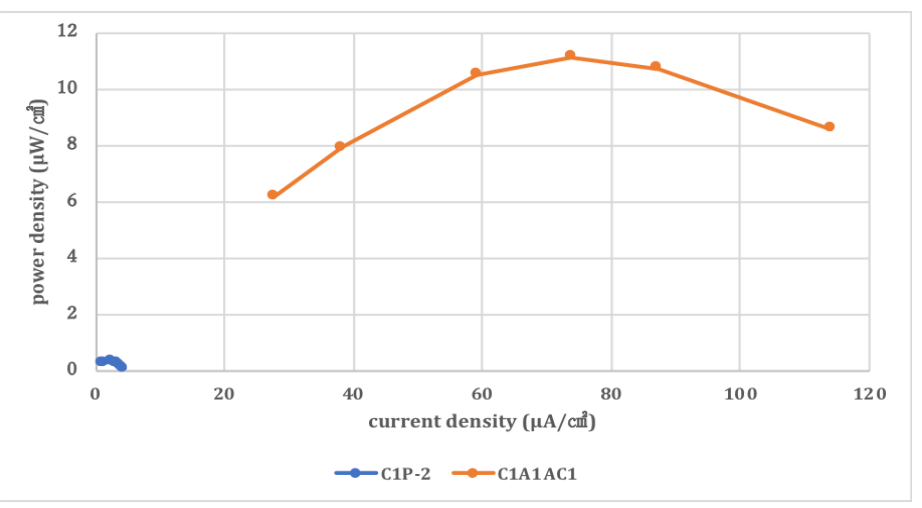

Fig. 9. Power density curves of the AC paper and hydrophilic CP

(2) Power density measurement using two kinds of AC paper with the thickness of $0.23 \mathrm{~mm}$ and $0.35 \mathrm{~mm}$ as the cathode electrodes.

As shown in Fig. 11, the maximum power densities using AC papers with thicknesses of $0.23 \mathrm{~mm}$ and $0.35 \mathrm{~mm}$ respectively were $11.1 \mu \mathrm{W} / \mathrm{cm}^{2}\left(73.8 \mu \mathrm{A} / \mathrm{cm}^{2}\right)$ and $15.9 \mu \mathrm{W} / \mathrm{cm}^{2}\left(88.1 \mu \mathrm{A} / \mathrm{cm}^{2}\right)$. The maximum power density using AC paper with thickness of $0.35 \mathrm{~mm}$ was about $43 \%$ higher than $0.23 \mathrm{~mm}$ because it has lager surface area. In the future, it is necessary to optimize the thickness of AC paper.

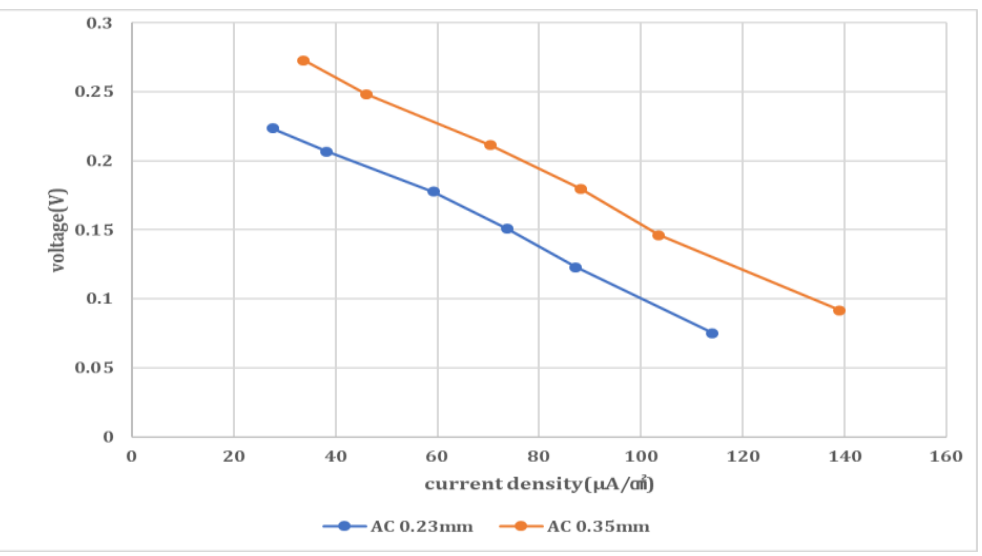

Fig. 10. Voltage and current density generated by the MFCs 


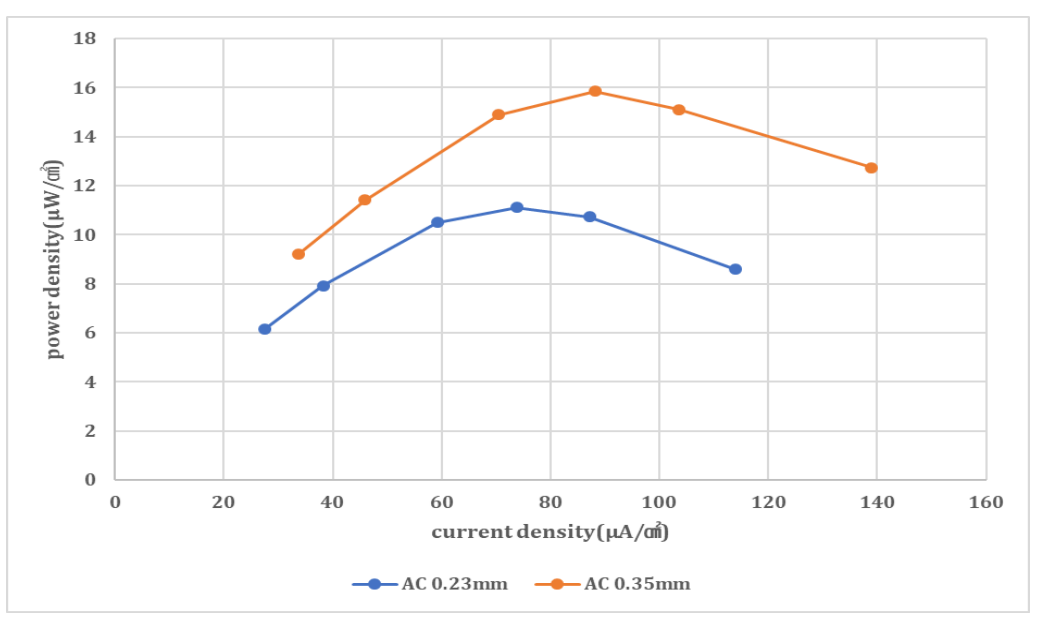

Fig. 11. Power density curves with two kinds of AC paper of different thicknesses

\section{CONCLUSION}

In this study, the performance of the AC paper was compared to the hydrophilic CP, which used commonly as anode electrode materials in MFCs, and the performance of AC paper with different thicknesses as cathode electrode materials were compared. SEM images and E. coli absorbance test showed that a large surface area of AC paper promotes biofilm adhesion. The MFC with the AC paper produced the maximum power density of $11.1 \mu \mathrm{W} / \mathrm{cm}^{2}$, which was much higher than that of the hydrophilic $\mathrm{CP}$. Moreover, we achieved $43 \%$ improvement in power density when AC paper with thicker thickness was used as the cathode electrode. Thus, the power generation was influenced by the surface area of the electrode materials and the internal resistance. The higher surface area helps to promote biofilm adhesion and reduce internal resistance. Furthermore, the cathode electrode having larger surface area made electrochemical reduction reaction to occur on the cathode surface more efficiently. These factors led to boost the power density output.

\section{ACKNOWLEDGMENTS}

We would like to thank Simon, Siraishi, and Suzaki for valuable suggestions. A part of this research was supported by AZUMI FILTER PAPER CO., Ltd.

\section{REFERENCES}

[1] H. Park, Y. H. Heo, P. Kim, and K. S. Nahm, "Direct electron transfer in E. coli catalyzed MFC with a magnetite/MWCNT modified anode," RSC Adv., vol. 3, no. 37, pp. 16665-16671, 2013.

[2] M. Mashkour and M. Rahimnejad, "Effect of various carbon-based cathode electrodes on the performance of microbial fuel cell," Biofuel Res. J., vol. 2, no. 4, pp. 296-300, 2015.

[3] M.-Y. Xi and Y.-P. Sun, "Preliminary study on E. coli microbial fuel cell and on-electrode taming of the biocatalyst," Guocheng Gongcheng Xuebao/The Chinese J. Process Eng., vol. 8, no. 6, pp. 1179-1184, 2008.

[4] F. Offei et al., "A viable electrode material for use in microbial fuel cells for tropical regions," Energies, vol. 9, no. 1, pp. $1-14,2016$.

[5] M. Rahimnejad, A. Adhami, S. Darvari, A. Zirepour, and S. E. Oh, "Microbial fuel cell as new technol ogy for bioelectricity generation: A review," Alexandria Eng. J., vol. 54, no. 3, pp. 745-756, 2015.

[6] N. Samsudeen, S. Chavan, T. K. Radhakrishnan, and M. Matheswaran, "Performance of microbial fuel cell using chemically synthesized activated carbon coated anode,” J. Renew. Sustain. Energy, vol. 8, no. 4, pp. 4-14, 2016.

[7] J. Hayashi, A. Kazehaya, K. Muroyama, and A. P. Watkinson, "Preparation of activated carbon from lignin by chemical activation," Carbon N. Y., vol. 38, no. 13, pp. 1873-1878, 2000.

[8] S. S. Manickam, U. Karra, L. Huang, N. N. Bui, B. Li, and J. R. McCutcheon, "Activated carbon nanofiber anodes for microbial fuel cells," Carbon N. Y., vol. 53, pp. 19-28, 2013.

[9] V. J. Watson, C. Nieto Delgado, and B. E. Logan, "Influence of Chemical and Physical Properties of Activated Carbon Powders on Oxygen Reduction and Microbial Fuel Cell Performance,” Environ. Sci. Technol., p. 130603162645003, 2013. 